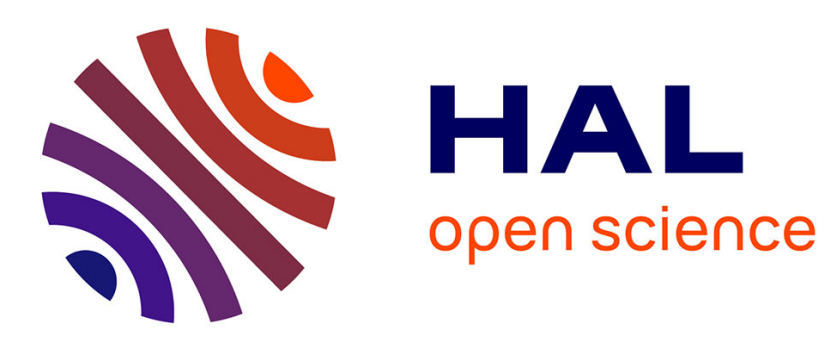

\title{
Vlasov simulations of plasma-wall interactions in a magnetized and weakly collisional plasma
}

\author{
S. Devaux, G. Manfredi
}

\section{To cite this version:}

S. Devaux, G. Manfredi. Vlasov simulations of plasma-wall interactions in a magnetized and weakly collisional plasma. Physics of Plasmas, 2006, 13, pp.083504. 10.1063/1.2244533 . hal-00206077

\section{HAL Id: hal-00206077 \\ https://hal.science/hal-00206077}

Submitted on 17 May 2021

HAL is a multi-disciplinary open access archive for the deposit and dissemination of scientific research documents, whether they are published or not. The documents may come from teaching and research institutions in France or abroad, or from public or private research centers.
L'archive ouverte pluridisciplinaire HAL, est destinée au dépôt et à la diffusion de documents scientifiques de niveau recherche, publiés ou non, émanant des établissements d'enseignement et de recherche français ou étrangers, des laboratoires publics ou privés. 


\title{
Vlasov simulations of plasma-wall interactions in a magnetized and weakly collisional plasma
}

\author{
S. Devaux ${ }^{\mathrm{a})}$ \\ Laboratoire de Physique des Milieux Ionisés et Applications, CNRS and Université Henri Poincaré, \\ F-54506 Vandoeuvre-les-Nancy, France \\ G. Manfredi ${ }^{\text {b) }}$ \\ Institut de Physique et Chimie des Matériaux de Strasbourg, UMR 7504 ULP-CNRS, \\ 23 rue du Loess, BP 43, F-67034 Strasbourg Cedex 2, France
}

(Received 6 April 2006; accepted 30 June 2006; published online 21 August 2006)

A Vlasov code is used to model the transition region between an equilibrium plasma and an absorbing wall in the presence of a tilted magnetic field, for the case of a weakly collisional plasma $\left(\lambda_{\mathrm{mfp}} \gg \rho_{i}\right.$, where $\lambda_{\mathrm{mfp}}$ is the ion-neutral mean-free path and $\rho_{i}$ is the ion Larmor radius). The phase space structure of the plasma-wall transition is analyzed in detail and theoretical estimates of the magnetic presheath width are tested numerically. It is shown that the distribution near the wall is far from Maxwellian, so that temperature measurements should be interpreted with care. Particular attention is devoted to the angular distribution of ions impinging on the wall, which is an important parameter to determine the level of wall erosion and sputtering. (C) 2006 American Institute of Physics. [DOI: 10.1063/1.2244533]

\section{INTRODUCTION}

The vast majority of plasmas produced in the laboratory are in contact with a material surface. Therefore, understanding the physical processes that are at play in plasma-wall interactions is a matter of paramount importance. In fusion devices, the surface can be either the material vessel that contains the plasma, or some ad hoc device (limiter or divertor) specifically designed to optimize the interaction with the charged particles. The scrape-off layer, i.e., the region of plasma directly in contact with the wall, can erode the surface and release high- $Z$ impurities, which migrate toward the bulk plasma and deteriorate its confinement. ${ }^{1}$ In low pressure plasmas, the understanding of plasma-wall interactions is even more fundamental, because treating a surface is often the very goal to achieve. Thus, the ion energy spectrum in front of the wall is a crucial factor for the quality of the surface treatment. Yet another research field where plasmawall interactions cannot be ignored is probe measurements. ${ }^{2}$ Probes are small metallic objects introduced in the plasma in order to measure certain macroscopic properties (e.g., density, electric current). As the probe's surface can disturb the plasma characteristics, its presence must be taken into account in order to interpret correctly the outcomes of a measurement.

It is well known that the transition region between an unmagnetized plasma at thermodynamic equilibrium and an unbiased and perfectly absorbing wall is composed of two different subregions: the Debye sheath (DS) and the collisional presheath $(\mathrm{CP})$. The DS, located just in front of the wall, is positively charged and tends to shield the negative bias of the wall. Bohm showed in his work ${ }^{3}$ that the DS stability requires a supersonic ion flow at the DS entrance

\footnotetext{
${ }^{a)}$ Electronic mail: Stephane.Devaux@1pmi.uhp-nancy.fr

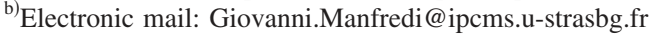

(a condition now known as the "Bohm criterion"). As the ion average velocity is generally smaller than the sound speed in the bulk plasma, an intermediate region is needed in order to increase the ion velocity up to the ion sound speed. This region, called the collisional presheath $(\mathrm{CP})$, is quasineutral and dominated by ion-neutral collisions. The unmagnetized plasma-wall transition was investigated theoretically and numerically in several works-see, for instance, Refs. 4-7.

When an oblique magnetic field is applied, ${ }^{8}$ the plasmawall transition is substantially modified. According to the theoretical study carried out by Ahedo, ${ }^{9}$ three fundamental length scales arise in the description of magnetized plasmawall interactions. As in the unmagnetized case, the electron Debye length $\left(\lambda_{\mathrm{D} e}\right)$ and the ion-neutral mean-free path $\left(\lambda_{\mathrm{mfp}}\right)$ determine the typical thickness of the DS and CP, respectively. The new important length scale is the ion Larmor radius $\left(\rho_{i}\right)$, which governs physical phenomena due to the magnetic field. For the special case where $\lambda_{\mathrm{D} e} \ll \rho_{i} \ll \lambda_{\text {mfp }}$, Ahedo showed that the plasma-wall transition is composed of three separate regions. In the vicinity of the wall, the DS is dominated by space-charge effects and only mildly affected by the presence of the magnetic field. Farther from the wall, the magnetic field forces the charged particles to follow the field lines, so that ions are mainly accelerated in the direction parallel to B. The crucial point is that Bohm's criterion should still be satisfied at the Debye Sheath Edge (DSE), where the ions' velocity normal to the wall should be at least equal to the acoustic velocity. Therefore, a new intermediate region (located between the DS and the CP) is needed in order to redirect the ionic flow toward the wall: this region is the magnetic presheath (MP), and its thickness is found to be of the order of the ion Larmor radius.

In the present paper, we investigate the magnetized plasma-wall interaction using a kinetic model that allows us to follow the ion dynamics along these three regions 


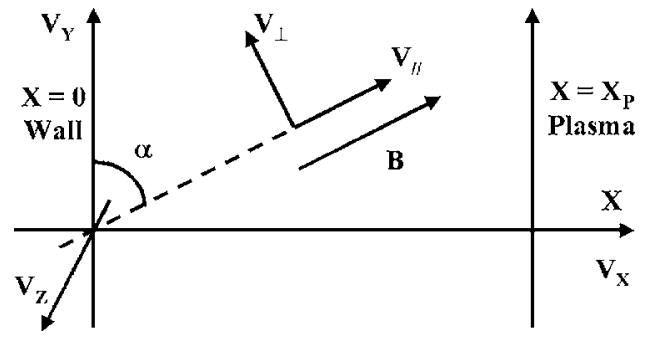

FIG. 1. Geometry of the plasma-wall transition. The wall lies in the $\mathrm{yOz}$ plane, at $x=0$, and the equilibrium plasma is located at $x \geq x_{p}$. The magnetic field $\mathrm{B}$, in the $\mathrm{xOy}$ plane, makes an angle $\alpha$ with the wall. Note that $v_{\perp}$ is defined as the component of the perpendicular velocity that lies in the $\mathrm{xOy}$ plane.

(CP, MP, and DS), from the bulk plasma to the wall. In the next section, we shall introduce the pertinent kinetic model and the related numerical techniques. Subsequently, several aspects of the plasma-wall transition will be illustrated through numerical simulations of the kinetic model.

\section{THEORETICAL MODEL AND NUMERICAL TECHNIQUES}

\section{A. Kinetic model}

Our aim in this work is to simulate the interactions between a plasma at thermodynamic equilibrium and a perfectly absorbing wall, when a tilted homogeneous magnetic field is applied. The geometry of the problem is given in Fig. 1. The unperturbed plasma is located at $x \geq x_{p}$ and the wall at $x=0$; the magnetic field lies in the xOy plane and makes an angle $\alpha$ with the Oy direction. As the wall is supposed to be infinite in the yOz plane, this physical system is invariant by translation in this plane: the study can thus be reduced to one dimension in space and three dimensions in velocity, yielding a "1D3V" problem.

The pioneering work of Chodura on magnetized plasmawall interactions ${ }^{8}$ did not take into account collisional effects. Subsequent studies have included ion-neutral collisions within a fluid model. ${ }^{10}$ However, a fluid model is not adequate to describe the presheath, because of the strong inhomogeneity in front of the DS and also because of the different orbit types present in the MP. More recent numerical studies were carried out with kinetic models, by making use of particle-in-cell (PIC) $\operatorname{codes}^{11}$ or by other phase-space techniques. $^{12,13}$ Here, we shall develop an Eulerian method to solve the relevant kinetic equation for the ion distribution function. With respect to PIC simulations, its main advantage is to considerably reduce the level of numerical noise, particularly in regions where the ion distribution is dilute, such as the DS. Though more accurate, Eulerian codes are, however, more demanding in terms of computation time and memory storage.

The model is based on the evolution of the ion distribution function $f_{i}(x, \mathbf{v}, t)$, which is governed by a modified Vlasov equation,

$$
\frac{\partial f_{i}}{\partial t}+v_{x} \frac{\partial f_{i}}{\partial x}+\frac{e}{m_{i}}(\mathbf{E}+\mathbf{v} \times \mathbf{B}) \cdot \frac{\partial f_{i}}{\partial \mathbf{v}}=-\nu\left(f_{i}-f_{0}\right),
$$

where $\mathbf{E}$ and $\mathbf{B}$ are the electric and magnetic fields, $x$ is the position coordinate normal to the wall, and $\mathbf{v}$ is the velocity vector. A generalized Bhatnagar-Gross-Krook (BGK) term has been added to the Vlasov equation in order to simulate the effects of ion-neutral collisions and ionization. The neutral distribution $f_{0}(v)$ is Maxwellian with temperature $T_{i 0}$ and density $n_{0}$. The BGK term tends to rebuild the equilibrium distribution $f_{0}(v)$ with a rate equal to $\nu=v_{\text {thi }} / \lambda_{\mathrm{mfp}}$, where $\lambda_{\text {mfp }}$ is the ion-neutral mean-free path, and $v_{\text {thi }}$ is the ion thermal velocity.

Here $f_{0}(v)$ also represents the ion distribution in the bulk plasma, where the ions are assumed to be at thermodynamic equilibrium with the neutrals, and therefore it is used as a boundary condition for $f_{i}$ at $x=x_{p}$. The wall, located at $x=0$, is assumed to be perfectly absorbing, so that ions can leave the system, but not reenter (zero incoming flux).

The electrons are assumed to be at thermodynamic equilibrium with temperature $T_{e}$. In order to obtain a selfconsistent set of equations, Eq. (1) is coupled to Poisson's equation:

$$
\frac{\partial^{2} \phi}{\partial x^{2}}=-\frac{e}{\epsilon_{0}}\left[n_{i}-n_{0} \exp \left(e \phi / k_{\mathrm{B}} T_{e}\right)\right],
$$

where $\int f_{i} \mathrm{~d} \mathbf{v}$ is the ion density, $\boldsymbol{\epsilon}_{0}$ is the vacuum dielectric constant, $k_{\mathrm{B}}$ is Boltzmann's constant, $n_{0}$ is the equilibrium density in the unperturbed plasma, and $\phi$ is the electric potential related to the electric field via the relation $E_{x}=$ $-\partial \phi / \partial x$. The following boundary conditions are adopted for the Poisson equation: (i) on the plasma side $\left(x=x_{p}\right)$, the potential is set to zero; (ii) at the wall $(x=0)$, a floating potential condition is assumed, given by the accumulation of electric charges on the wall. The floating potential is computed by integrating Ampère's equation on the wall:

$$
\frac{\partial E_{x}}{\partial t}=-\frac{e}{\epsilon_{0}}\left(j_{i}-j_{e}\right) .
$$

The ion flux toward the wall is given by $j_{i}=\int v_{x} f_{i} d v$. The electron flux is estimated by assuming that the electron velocity distribution is half-Maxwellian on the wall, which yields

$$
j_{e}(0, t)=n_{0}\left(\frac{k_{\mathrm{B}} T_{e}}{2 \pi m_{e}}\right)^{1 / 2} \exp \left(\frac{e \phi(0)}{k_{\mathrm{B}} T_{e}}\right) .
$$

We also assumed that, under the physical conditions considered for our simulations, secondary electron emission at the wall is negligible. We are aware that one should be cautious with the latter hypothesis, as a small amount of secondary electrons can affect the structure of the plasma-wall transition. $^{14}$

\section{B. Numerical methods-Nonuniform grid}

The numerical method we use to solve the VlasovPoisson set of equations is based on a time-splitting technique developed by Cheng and Knorr. ${ }^{15}$ This method 
amounts to splitting the Vlasov equation into a sequence of simpler equations, which are then solved one after the other (see Ref. 16 for further details):

$$
\begin{aligned}
& \frac{\partial f_{i}}{\partial t}+v_{x} \frac{\partial f_{i}}{\partial x}=0, \\
& \frac{\partial f_{i}}{\partial t}+\frac{e}{m_{i}}(\mathbf{E}+\mathbf{v} \times \mathbf{B}) \cdot \frac{\partial f_{i}}{\partial \mathbf{v}}=0, \\
& \frac{\partial f_{i}}{\partial t}=-\nu\left(f_{i}-f_{0}\right) .
\end{aligned}
$$

Equations (5) and (6) possess exact solutions, which consist of a constant drift of the distribution function in the $x$ direction, or in each of the three velocity directions. Equation (7) corresponds to a "mixing" between $f_{i}$ and the equilibrium distribution $f_{0}$ and also has an obvious analytical solution. Usually, the drifts related to Eqs. (5) and (6) do not correspond to an entire number of grid steps, so that some kind of interpolation needs to be performed: in the present work; we make use of a finite-volume scheme. ${ }^{17}$ The resulting numerical scheme is only first order accurate in time, but this limitation is not too serious, as we are mainly interested in the steady state of the system.

Poisson's equation must be solved immediately before Eq. (6) in order to obtain the electric potential and the electric field. The presence of the Boltzmann factor renders the equation nonlinear, so that Poisson's equation must be solved with an iterative method whose details can be found in Ref. 16.

Our aim in this work is to simulate the plasma-wall transition in the special case where

$$
\lambda_{\mathrm{D} e} \ll \rho_{i} \ll \lambda_{\mathrm{mfp}} .
$$

As the various scale lengths differ considerably, an obvious numerical problem arises: if the grid step is small enough to correctly describe the DS, then too many points are wasted to mesh the entire CP. Typically, the grid step should be around $\lambda_{\mathrm{D} e}=\sqrt{T_{e} / T_{i}} \lambda_{\mathrm{D} i}$ in the DS, with a simulation box approximately $15000 \lambda_{\mathrm{D} i}$ long in order to contain the whole CP. For a temperature ratio $T_{e} / T_{i}=10$, this yields roughly 5000 grid points in the $x$ direction. As we take 60 grid points for each velocity direction, a standard simulation with a uniform mesh would require more than a $10^{9}$ grid point.

To decrease the number of grid points, we resort to a nonuniform grid by transforming the "real" spatial coordinate $x$ into a new space coordinate $s$ :

$$
\begin{aligned}
& d x=g(s) d s, \\
& g(s)=\Delta x_{1}+\frac{\Delta x_{2}-\Delta x_{1}}{2}\left\{1+\tanh \left[c\left(s-s_{0}\right)\right]\right\},
\end{aligned}
$$

where $\Delta x_{1}$ and $\Delta x_{2}$ are two constant grid steps, and $c$ and $s_{0}$ are two coefficients that control the shape of the grid function $g(s)$.

Using a constant step $\Delta s$ in the transformed coordinate, we get a nonuniform step in the original $x$ variable. By carefully choosing the grid function (see Fig. 2), we can obtain a

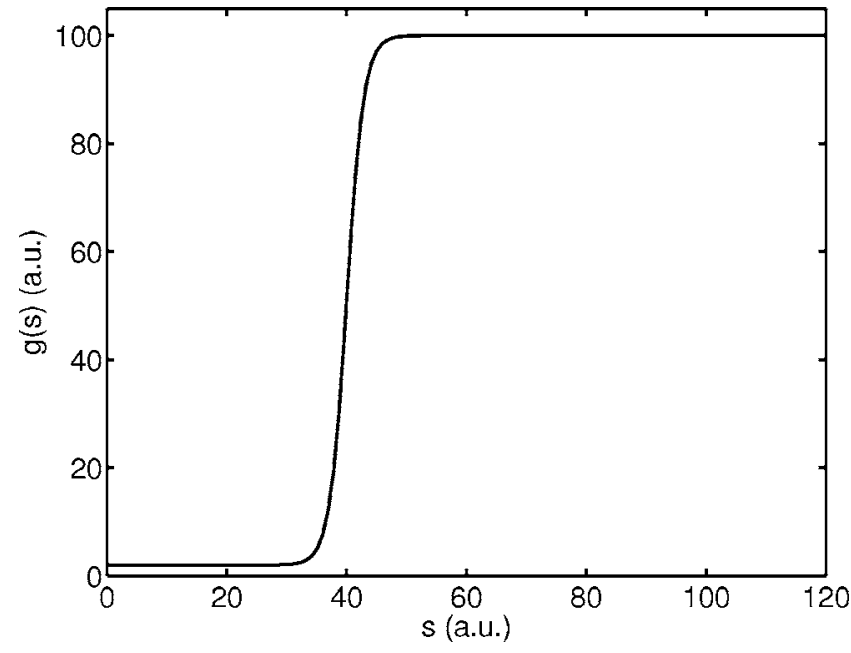

FIG. 2. Grid function $g(s)$ for the nonuniform mesh. The corresponding grid steps $\Delta x$ are equal to $2 \lambda_{\mathrm{D} i}$ near the wall and $100 \lambda_{\mathrm{D} i}$ in the collisional presheath.

small grid step $\Delta x_{1}$ in the DS near the wall and a larger one $\Delta x_{2}$ in the presheath. Typical values are $\Delta x_{1}=2 \lambda_{\mathrm{D} i}$, $\Delta x_{2}=100 \lambda_{\mathrm{D} i}, s_{0}=40 \lambda_{\mathrm{D} i}$ and $c=0.3 \lambda_{\mathrm{D} i}^{-1}$. Note that $s_{0}$ and $c$ represent, respectively, the position of the transition between the small and the large grid steps, and the steepness of the shape function $g(s)$.

The above change of variables is reflected in the Vlasov and Poisson equations (1) and (2), which become, respectively,

$$
g \frac{\partial f_{i}}{\partial t}+v_{x} \frac{\partial f_{i}}{\partial s}+g \frac{e}{m_{i}}(\mathbf{E}+\mathbf{v} \times \mathbf{B}) \cdot \frac{\partial f_{i}}{\partial \mathbf{v}}=-g \nu\left(f_{i}-f_{0}\right),
$$

$$
\frac{\partial^{2} \phi}{\partial s^{2}}-\frac{g^{\prime}}{g} \frac{\partial \phi}{\partial s}=-\frac{e}{\epsilon_{0}}\left[n_{i}-n_{0} \exp \left(e \phi / k_{\mathrm{B}} T_{e}\right)\right]
$$

Because we are only interested in the steady state $\left(\partial f_{i} / \partial t\right.$ $=0$ ), the factor $g(s)$ in front of the time derivative in Eq. (11) can be omitted: the resulting equation has the same stationary solutions and the same formal structure as Eq. (1), so that the same numerical method can be used to solve it. Of course, this modified Vlasov equation cannot be used to study time-dependent problems, as it is not equivalent to the correct Vlasov equation, except for the steady states.

The modified Poisson's equation (12) is again solved using an iterative technique based on centered finite differences, which leads to a tridiagonal matrix to be inverted. The matrix inversion can yield a numerical instability if the following condition is not satisfied:

$$
1-\frac{g^{\prime}(s)}{g(s)} \frac{\Delta s}{2}>0,
$$

where $\Delta s$ is the grid step for the $s$ coordinate and $g^{\prime}(s)=d g / d s$. This condition is satisfied when the transition between the two grid steps $\Delta x_{1}$ and $\Delta x_{2}$ is sufficiently smooth. Indeed, Eq. (13) can be rewritten as $\Delta s<2 L_{g}$, where $L_{g}=g / g^{\prime}$ is the typical scale length of variation of the grid 
shape function. This condition can be kept under control by carefully choosing the free parameters $c$ and $s_{0}$.

In summary, the use of a nonuniform grid allowed us to reduce the number of grid points in the $x$ direction to $N_{x} \simeq 150$, which represents a gain of more than one order of magnitude compared to a regular mesh.

\section{Physical conditions}

In order to simulate realistic physical conditions, four dimensionless parameters can be adjusted. Two of them have already been mentioned: the ion-neutral collision frequency $\nu$ (normalized to the ion plasma frequency $\omega_{p i}$ ) and the angle $\alpha$ between the magnetic field and the wall. The two other parameters are $\tau=T_{e} / T_{i 0}$, the ratio of the electron to the ion temperature in the bulk plasma, and $\omega=\omega_{c i} / \omega_{p i}$, the ratio of the ion cyclotron frequency to the ion plasma frequency. In the present work, our aim is to describe the plasma-wall transition in low-pressure plasma experiments, such as the MIRABELLE device based at the University of Nancy, France. ${ }^{18}$ Typical physical parameters in MIRABELLE (which works mainly with argon plasmas) are $n_{0}=2$ $\times 10^{15} \mathrm{~m}^{-3}, T_{e}=2 \mathrm{eV}, T_{i 0}=0.03 \mathrm{eV}, \omega_{c i}=10^{4}-10^{5} \mathrm{rad} / \mathrm{s}$, $\omega_{p i}=10^{7} \mathrm{rad} / \mathrm{s}$, and $\nu_{i-n}=10^{3} \mathrm{~Hz}$. The neutrals are supposed to be at the same temperature as the ions, which is usual for low-pressure plasma devices. This yields the following dimensionless values: $\omega \approx 10^{-3}-10^{-2}, \tau \approx 60$, and $\nu \approx 10^{-4}$.

In our simulations, we used values in the following ranges: $\nu=10^{-3}-10^{-2}, \quad \tau=5-35, \omega=5 \times 10^{-3}-10^{-1}$, and $\alpha=20^{\circ}, 40^{\circ}$, and $60^{\circ}$. These values still allow us to respect the ordering of Eq. (8). For all cases, the electron-to-ion mass ratio is that of an argon plasma, $m_{e} / m_{i}=1.351 \times 10^{-5}$. It must be noted that the above dimensionless parameters are relatively close to those found in the scrape-off layer (SOL) of tokamak devices, although the temperature ratio in a SOL plasma is generally of order unity.

\section{NUMERICAL RESULTS}

In this section, we present results from our numerical simulations. Each run begins with a spatially homogeneous plasma at thermodynamic equilibrium (ion Maxwellian distribution at temperature $T_{i 0}$ ) in the whole transition region between $x=0$ and $x=x_{p}$. Then, the plasma is allowed to evolve according to the Vlasov-Poisson system until it reaches a(n) (inhomogeneous) steady state. The run is stopped when the spatial profiles of physically relevant quantities (e.g., density, average velocity) do not evolve significantly anymore. As an example, the ion and electron densities for a typical run are plotted in Fig. 3. As expected, the plasma is quasineutral in the $\mathrm{CP}$ and MP, whereas a charge separation is observed in the DS near the wall.

\section{A. Debye sheath}

The Bohm criterion tells us that the ion velocity normal to the wall should be at least equal to the ion sound speed $c_{s}=\sqrt{k_{\mathrm{B}}\left(T_{e}+T_{i 0}\right) / m_{i}}$ at the DS entrance. The original criterion was derived for a simple situation where the ions are cold and the plasma is collisionless and unmagnetized. A

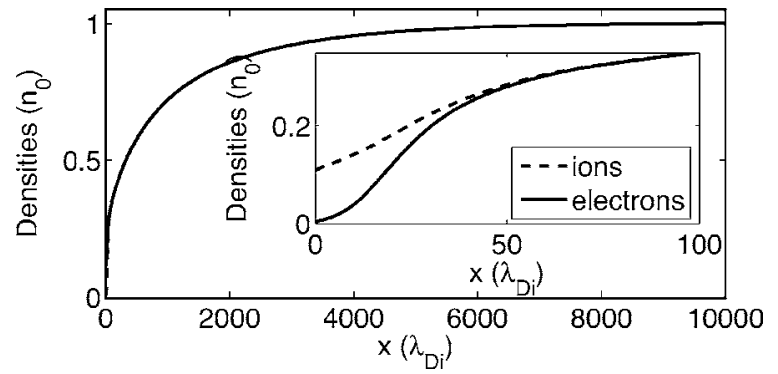

FIG. 3. Ion and electron density profiles along the plasma-wall transition. The zoom shows the positively charged layer in the DS.

kinetic version of Bohm's criterion was later derived, in which the average velocity $\left\langle v_{x}\right\rangle$ is replaced by $\left\langle v_{x}^{-2}\right\rangle^{-1 / 2}$. However, the kinetic criterion does not work well for collisional plasmas, as the ion distribution at the DS entrance does not necessarily vanish at $v_{x}=0$.

For a magnetized plasma, full kinetic simulations of the entire transition region showed that the Bohm criterion is not verified inside the DS. ${ }^{19}$ In order to check this point, we show the dependence of the Mach number $M_{x}=\left|\left\langle v_{x}\right\rangle\right| / c_{s}$ on the intensity and angle of incidence of the magnetic field (Fig. 4). The DS entrance is defined as the point where the charge separation $n_{i}-n_{e}$ is equal to $0.01 n_{0}$. The result is that Bohm's criterion is indeed not satisfied when the magnetic field is large and its incidence grazing, in agreement with Ref. 19.

\section{B. Magnetic presheath}

The spatial extension of the MP is determined by a competition between the magnetic field, which tries to keep ions traveling along the field lines (as in the $\mathrm{CP}$ ), and the electric field, which tends to reorient them along the direction normal to the wall (as in the DS). The MP is the intermediate region where these two effects are of the same order of magnitude.

Several theoretical and numerical studies have been carried out on the MP. In particular, Chodura ${ }^{8}$ provided the following expression for the spatial extension of the MP:

$$
\lambda_{\mathrm{MP}}^{\mathrm{th}} \approx \sqrt{6} \cos \alpha \frac{\tau^{1 / 2}}{\omega} \lambda_{\mathrm{D} i} .
$$

This expression can be derived by projecting the Larmor radius on the direction normal to the wall. Here $\lambda_{\mathrm{MP}}$ repre-

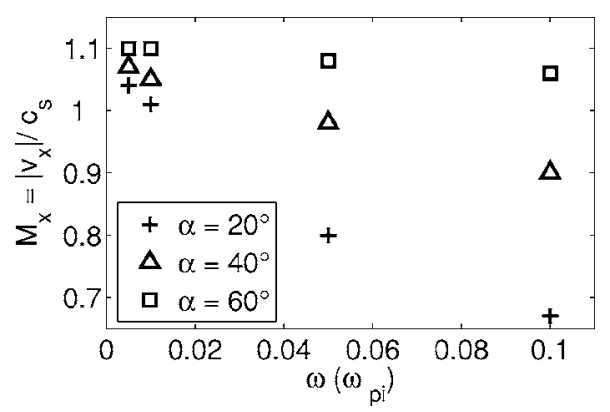

FIG. 4. Mach number at the DS entrance, for various values of the magnetic field intensity $\omega$ and inclination $\alpha$. The collision rate is $\nu=10^{-3}$ everywhere. 


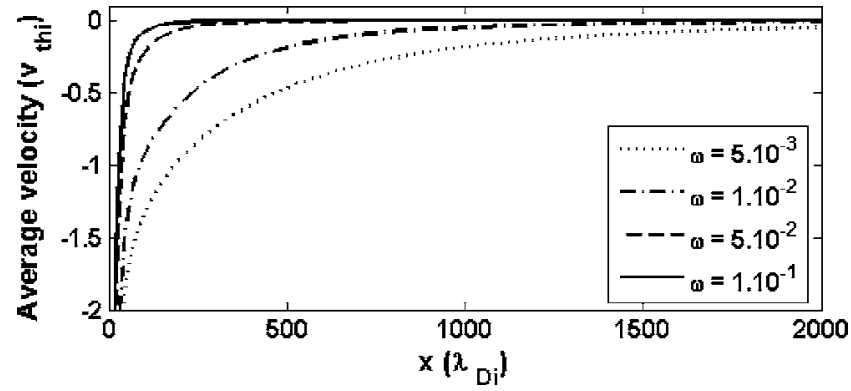

FIG. 5. The average perpendicular velocity for several values of $\omega$, with $\alpha=40^{\circ}, \tau=10$, and $\nu=10^{-3}$.

sents the distance where the orbits of particles with perpendicular velocity equal to $v_{\text {th } i}$ intersect the wall and can thus be collected. The origin of the factor $\sqrt{6}$ is less clear, but it was observed by Chodura in his numerical studies and appears to be consistent with our simulations as well. An experimental verification of Eq. (14) was performed in Ref. 20.

The above expression for the thickness of the MP is a good test for the simulations: indeed, the behavior of $\lambda_{\mathrm{MP}}$ with the various physical parameters $(\tau, \alpha$, and $\omega)$ can be verified. In order to perform such tests, we need a reliable procedure to determine $\lambda_{\mathrm{MP}}$ from the simulation results. As we shall see later, the main influence of the magnetic field in the $\mathrm{CP}$ is to guide the ion acceleration along the field lines, without affecting the motion in the perpendicular direction. Therefore, the magnetic presheath edge (MPE) can be defined as the point where the ion flux starts to be redirected toward the wall, i.e., the point where the average perpendicular (to B) velocity begins to deviate from the zero value that it has in the CP. Such a definition was introduced earlier by Gunn $^{21}$ and is consistent with the theoretical expression of Eq. (14). Other authors ${ }^{1,5,6}$ define the MPE as the point where the parallel ion velocity becomes sonic. The relationship between these two approaches will be illustrated in the next paragraphs.

Figure 5 shows the profile of the average perpendicular velocity $\overline{v_{\perp}}$ (defined in Fig. 1) in the MP and part of the CP, for several values of the magnetic field intensity $\omega$. Here $\overline{v_{\perp}}$ is zero in the bulk plasma and then increases first slowly, then more abruptly, near the wall. In order to obtain a workable criterion to extract $\lambda_{\mathrm{MP}}$ from the numerical results, one simulation (with $\omega=0.1, \alpha=40^{\circ}, \tau=10$, and $\nu=10^{-3}$ ) has been chosen as a reference. For this run, we assume the location of the MPE to be at $x=\lambda_{\mathrm{MP}}^{\mathrm{th}}$ and compute the value of $\overline{v_{\perp}}$ at this location. This value of $\overline{v_{\perp}}$ is then used, for the remaining simulations, as the definition of the MPE.

Figure 6(a) shows the dependence of the MP length on the angle of incidence $\alpha$ of the magnetic field, for $\tau=10, \nu$ $=10^{-3}$, and different values of $\omega$. The lines represent the theoretical result given by Eq. (14) and the points are numerical estimations of $\lambda_{\mathrm{MP}}$ obtained with the method described in the previous paragraph. The error bars represent the grid step at the location of the MPE. There is a relatively good agreement between the numerical results and the theoretical estimations. The agreement is better when $\omega$ is small. Cases where $\omega=0.100$ are the worst, with an overestimation of about $35 \%$. Such a discrepancy comes from the limitations of the theoretical model rather than from numerical errors. Indeed, in these cases the thickness of the MP is of the same order as the DS (about $60 \lambda_{\mathrm{D} i}$ ) and thus the plasmawall transition can no longer be clearly divided into three separate regions. This regime was also studied theoretically by Ahedo, ${ }^{9}$ but it is more complex and does not yield theoretical predictions that are easily comparable to the simulations. Our study will thus be limited to cases where the three regions (CP, MP, and DS) are clearly identified.

The behavior of $\lambda_{\mathrm{MP}}$ with $\tau$-the electron to ion tem-

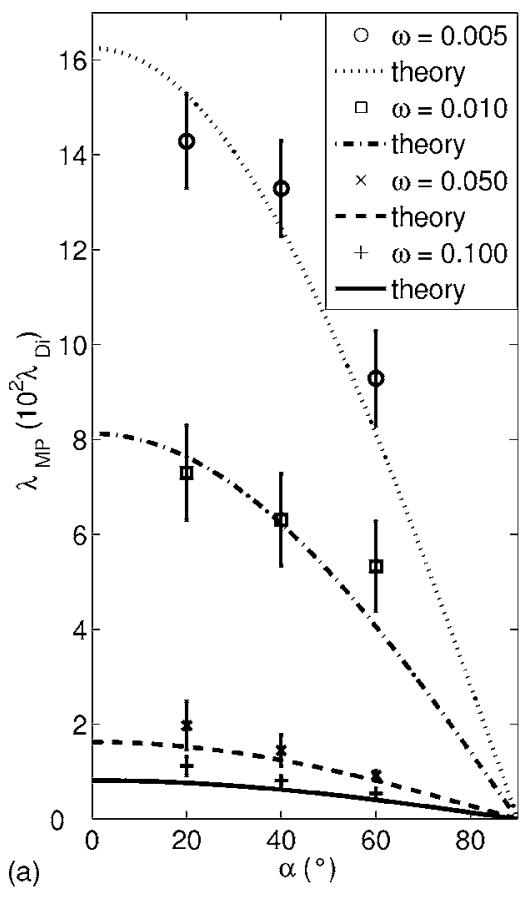

(b)
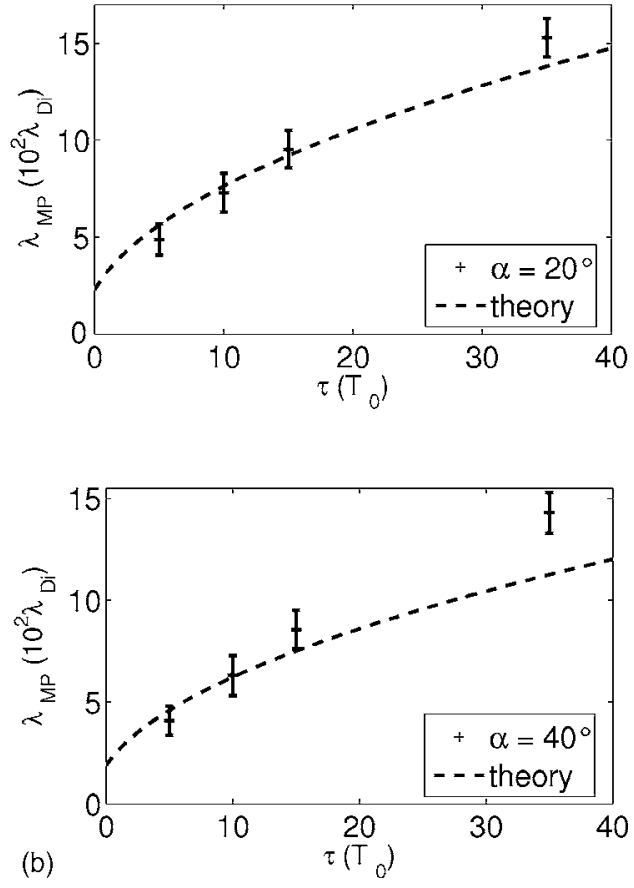

FIG. 6. Dependence of the MP length (a) with respect to $\alpha$ and $\omega$, for $\tau=10$ and $\nu=10^{-3}$; (b) with respect to $\tau$ and $\alpha$, for $\omega=0.010$ and $\nu=10^{-3}$. Points represent simulation results, whereas lines represent the theoretical estimation of Eq. (14) 

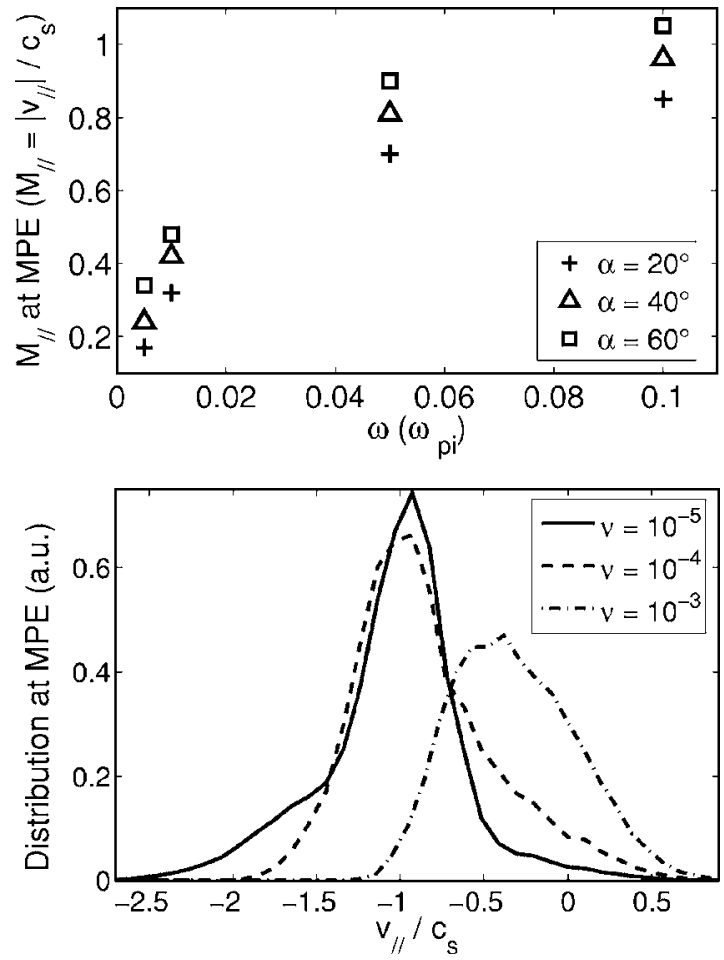

FIG. 7. Top: Parallel Mach number at the MPE, for $\nu=10^{-3}$ and various values of the magnetic field intensity $\omega$ and inclination $\alpha$. Bottom: Parallel velocity distribution for three values of the collision rate, and $\omega=0.01$, $\alpha=40^{\circ}$.

perature ratio-is given in Fig. 6(b), for $\alpha=20^{\circ}$ and $40^{\circ}$, and $\nu=10^{-3}$. A good agreement with theory is also obtained in this case, except for the runs at very low ion temperature $(\tau=35)$, which lie slightly off the theoretical estimate. The general trend is, however, respected.

Chodura ${ }^{8}$ has suggested that the ion parallel velocity must become sonic at the entrance of the MP (a condition now known as the Bohm-Chodura criterion). In Fig. 7 (top), we plot the parallel Mach number as a function of the magnetic field strength and angle of incidence. It appears that the Bohm-Chodura criterion is satisfied only for strong magnetic fields. It is less sensitive to the angle of incidence, although it is clear that the criterion is more easily satisfied for large values of $\alpha$. We can explain this effect as a result of the competition between the magnetic field and the collisions: the latter tend to restore the equilibrium distribution and thus to reduce the average parallel velocity below the sound speed. This is shown in Fig. 7 (bottom), where it is clear that the average parallel velocity at the MPE converges to the sound speed when the collision rate is reduced. In summary, we have compared two definitions of the MPE, as (a) the point where $\left\langle v_{\|}\right\rangle$becomes sonic; and (b) the point where the ions are redirected toward the wall (i.e., $\left\langle v_{\perp}\right\rangle$ becomes appreciable). They are equivalent (and in agreement with the theoretical estimate) when collisions are negligible compared to magnetic effects. In contrast, when collisions are important, the definition based on $\left\langle v_{\perp}\right\rangle$ still agrees with the theoretical expression of Eq. (14), whereas the Bohm-Chodura criterion is not satisfied (i.e., the parallel Mach number does not attain unity at the MPE).

\section{Phase-space distributions}

The ion phase-space distribution function $f_{i}$ contains all the information about the ion population and it is useful to inspect its modifications from the bulk plasma to the wall. Plots of the velocity distributions are rarely found in the existing literature, as most kinetic results were obtained by means of PIC simulations, which allow limited resolution in the phase space. A notable exception are the recent results by Sharma, ${ }^{13}$ which were obtained with a mesh-based code, although rather different from ours. The alternative method employed by Daube and Riemann ${ }^{12}$ samples the distribution function on a finite number of characteristics, yielding a highly singular distribution that is hardly comparable to our smooth results.

For ease of representation, we show two dimensional (2D) projections of the distribution function in velocity space, at different spatial locations within the plasma-wall transition. The projections are either in the $\mathrm{xOy}$ plane (where the magnetic field lies) or in the $\mathrm{xOz}$ plane (where the $\mathbf{E} \times \mathbf{B}$ drift can be observed).

For comparison, we first display the results for an unmagnetized plasma (Fig. 8), which also apply to a magnetized case with normal incidence $\left(\alpha=90^{\circ}\right)$. We show contour plots of $f_{i}$ in the $\left(v_{x}, v_{y}\right)$ plane along the plasma-wall transition, for $\tau=10$ and $\nu=10^{-3}$. In Fig. 8(a), i.e., in the bulk plasma, $f_{i}$ is given by a Maxwellian distribution with zero average velocity. In the $\mathrm{CP}$ [Fig. 8(b)], the distribution core drifts slightly along the direction normal to the wall. As expected, the ion acceleration is much stronger in the DS [Fig. 8(c)].

Figure 9 shows the evolution of $f_{i}$ in the $\left(v_{x}, v_{y}\right)$ plane, for a typical magnetized case with $\alpha=40^{\circ}, \omega=0.010, \tau=10$, and $\nu=10^{-3}$. The straight lines indicate the directions parallel
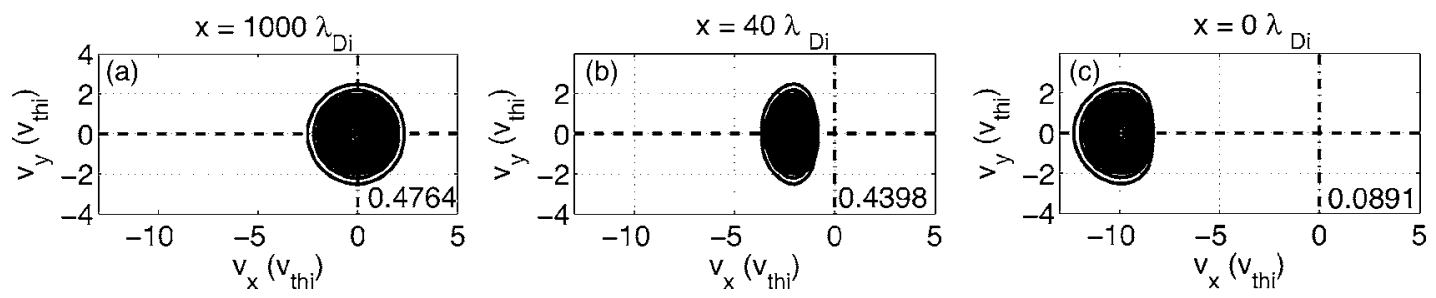

FIG. 8. Contours of the ion distribution function $f_{i}$ in the $\left(v_{x}, v_{y}\right)$ plane for an unmagnetized case, $\tau=10$ and $\nu=10^{-3}$. The distribution function is divided into 20 isodensity contours, the maximum value of $f_{i}$ being given at the lower right corner of each plot. (a) $x=1000 \lambda_{\mathrm{D} i}$ (bulk plasma); (b) $x=40 \lambda_{\mathrm{D} i}$ (DSE); and (c) $x=0$ (wall). 

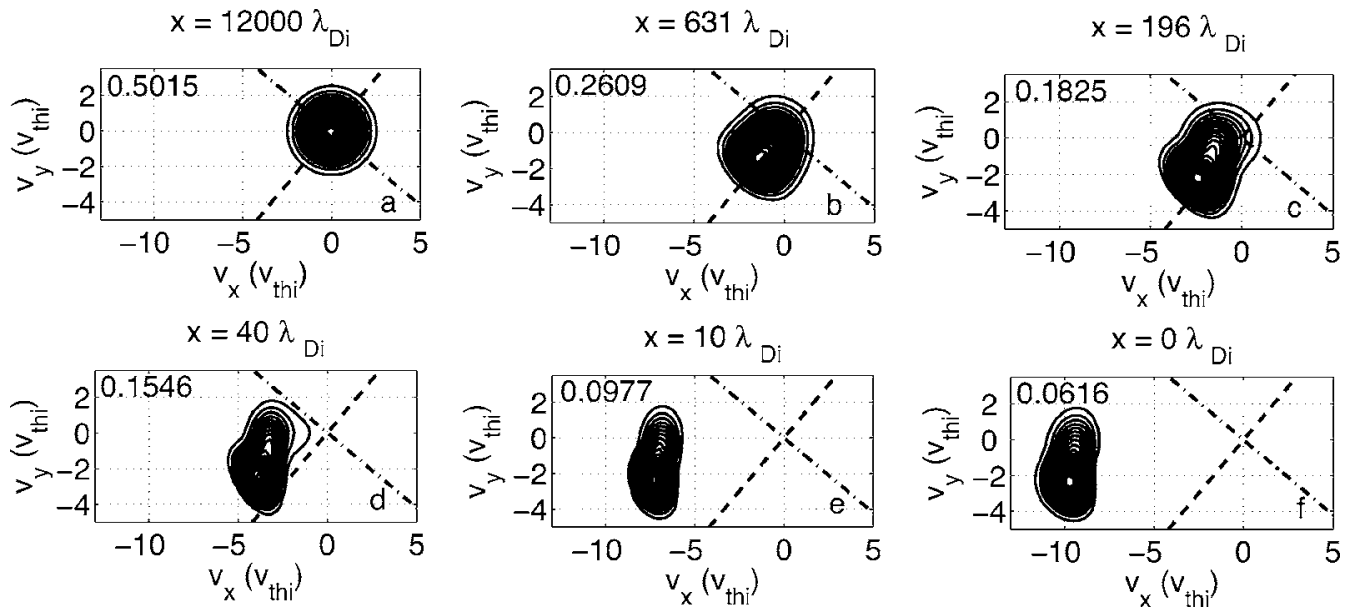

FIG. 9. Contours of the ion distribution function in the $\left(v_{x}, v_{y}\right)$ plane for a magnetized case, with $\alpha=40^{\circ}, \omega=0.010, \tau=10$, and $\nu=10^{-3} ; f_{i}$ is divided into 20 isodensity contours, the maximum value of $f_{i}$ being given at the top left corner of each plot. (a) $x=12000 \lambda_{\mathrm{D} i}$ (bulk plasma); (b) $x=631 \lambda_{\mathrm{D} i}(\mathrm{CP})$; (c) $x=196 \lambda_{\mathrm{D} i}$ (MPE); (d) $x=40 \lambda_{\mathrm{D} i}$ (DSE); (e) $x=10 \lambda_{\mathrm{D} i}$ (DS); and (f) $x=0$ (wall).

and perpendicular to the magnetic field. The value at the top left corner of each figure indicates the maximum of distribution at the location given in each caption; the distribution is then divided into 20 isodensity contours evenly spread between zero and its maximum.

In the bulk plasma [Fig. 9(a)], $f_{i}$ is still assumed to be a nondrifting Maxwellian. As expected, in the $\mathrm{CP} f_{i}$ drifts along the magnetic field lines. At the MPE [Fig. 9(b)], this effect is clearly visible: the ion distribution is centered on a finite parallel velocity, whereas the average perpendicular velocity is still zero. Here, the distribution function starts to be modified in the direction perpendicular to $\mathbf{B}$ : the ion flux is reoriented toward the wall in order to satisfy Bohm's criterion. This effect occurs within the MP [Fig. 9(c)] and is almost complete at the DS edge [Fig. 9(d)]. Finally, in the DS, the ions are strongly accelerated toward the wall by the electric field [Figs. 9(e) and 9(f)].

The evolution of $f_{i}$ in the $\left(v_{y}, v_{z}\right)$ plane can be followed in Fig. 10. In this plane, the ion distribution is less distorted and is seen to undergo two drifts. First, in the CP, the distribution drifts in the $v_{y}$ direction: this is simply the projection on the $v_{y}$ axis of the parallel drift observed in Fig. 9. Second, there is a drift in the $v_{z}$ direction that can be identified as the $E \times B$ drift (which is indeed parallel to the $z$ axis). More details on the latter effect will be provided in the next section.
The above analysis of the phase space portraits clarifies the role of the magnetic field on the plasma-wall transition. The ions are first accelerated in the $\mathrm{CP}$ along the magnetic field lines; in the MP, their velocities are redirected toward the wall; and finally, in the DS, they are strongly accelerated in the direction normal to the wall. These results are in agreement with the $2 \mathrm{D}$ phase-space plots recently published by Sharma. ${ }^{13}$

\section{Temperature and average velocity}

For laboratory plasmas in contact with a wall, the density and the temperature are crucial quantities to be measured. However, though measuring the plasma density is a relatively straightforward task, the interpretation of temperature diagnostics may present some ambiguity. Strictly speaking, the temperature is only defined for a population of particles with a Maxwellian distribution: the presence of walls distorts (often dramatically) the velocity distribution leading to non-Maxwellian profiles, so that the very concept of temperature may be meaningless. Of course, a temperature can always be defined from the velocity distribution in the usual way:
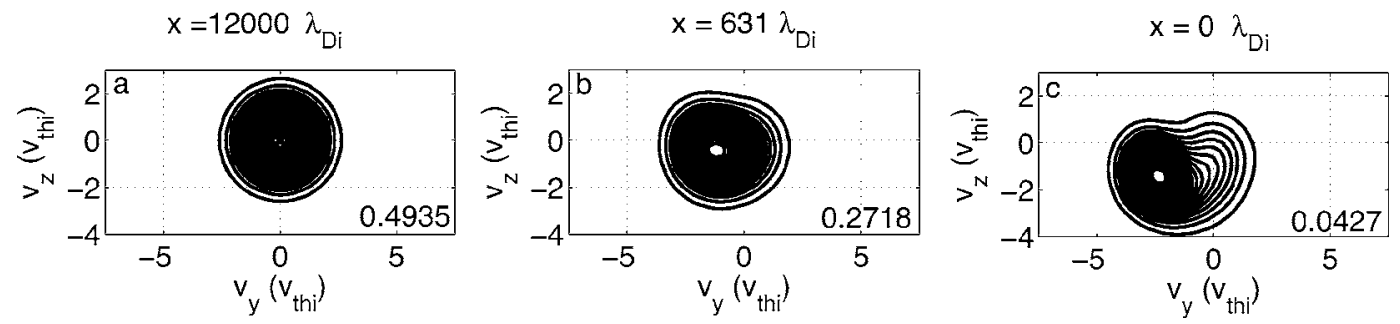

FIG. 10. Contours of the ion distribution function in the $\left(v_{y}, v_{z}\right)$ plane for a magnetized case, with $\alpha=40^{\circ}, \omega=0.010, \tau=10$, and $\nu=10^{-3} ; f_{i}$ is divided into 20 isodensity contours, the maximum value of $f_{i}$ being given at the lower right corner of each plot. (a) $x=12000 \lambda_{\mathrm{D} i}$ (bulk plasma); (b) $x=631 \lambda_{\mathrm{D} i}$ (MPE); and (c) $x=0$ (wall). 

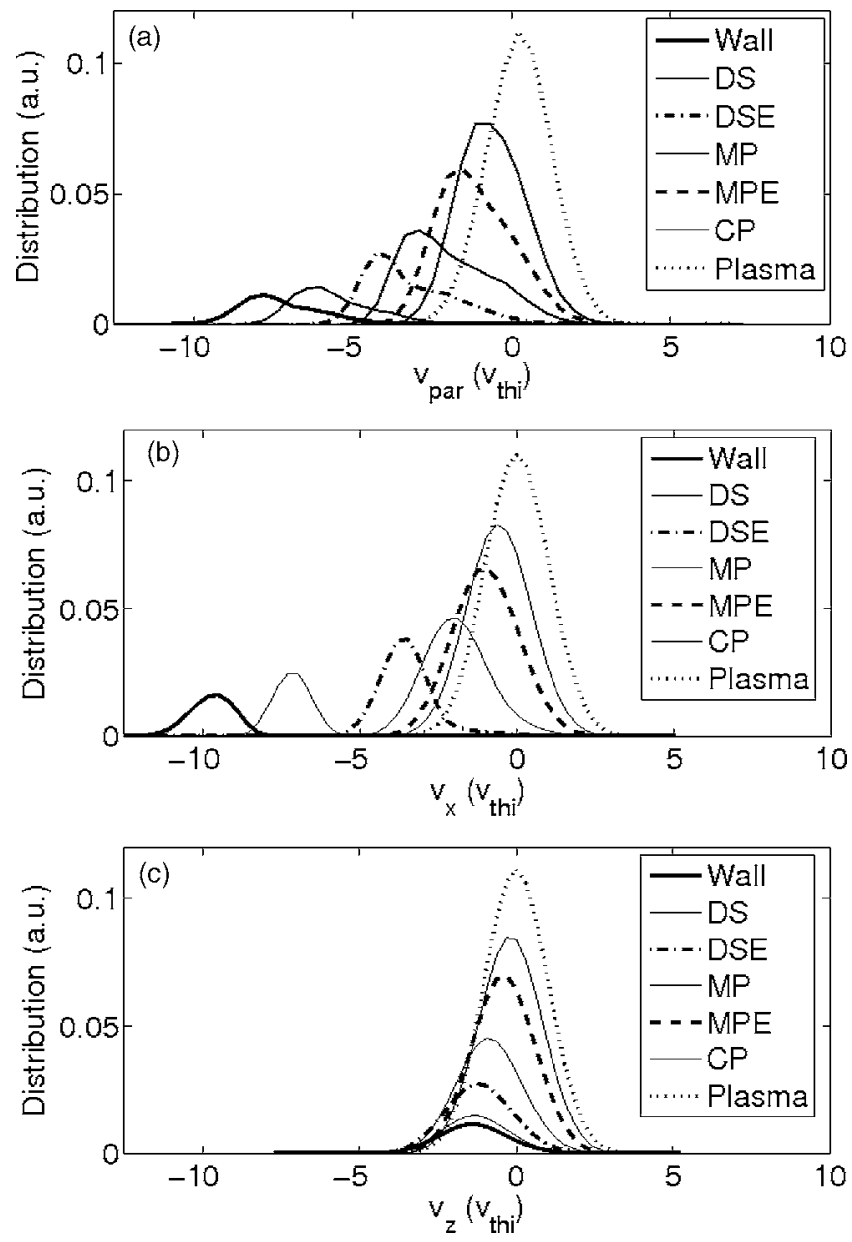

FIG. 11. 1D profiles of the ion velocity distribution, taken at different spatial positions, for $\alpha=40^{\circ}, \omega=0.010, \tau=10$, and $\nu=10^{-3}$ : (a) parallel velocity; (b) velocity normal to the wall; (c) velocity along the $z$ direction.

$$
T_{j}(x)=\int f_{i}(x, \mathbf{v})\left(v_{j}-\left\langle v_{j}\right\rangle\right)^{2} d \mathbf{v},
$$

where the subscript $j$ stands for the various directions (e.g., $x, y$, or parallel) along which we want to compute the temperature. Indeed, in a magnetized plasma, we do not necessarily expect perfect isotropy, so that the temperature can be different along different directions.

In order to interpret correctly the temperature profiles issued from the simulations, it is useful to plot 1D projections of the ion velocity distribution, particularly along the directions parallel to the magnetic field [Fig. 11(a)] and normal to the wall [Fig. 11(b)]. The distribution in the $z$ direction is also plotted in Fig. 11(c).

The first observation is that these three directions do not present the same velocity profiles. The $v_{\|}$distribution is more distorted and-between the MPE and the wall-develops a long tail extending for several ion thermal velocities. These profiles are similar to those obtained along the normal direction in the case of an unmagnetized transition (see Fig. 12). As a consequence of the distortion of the $v_{\|}$distribution, the parallel temperature obtained from Eq. (15) may overestimate the "real" temperature. This is apparent in the tempera-

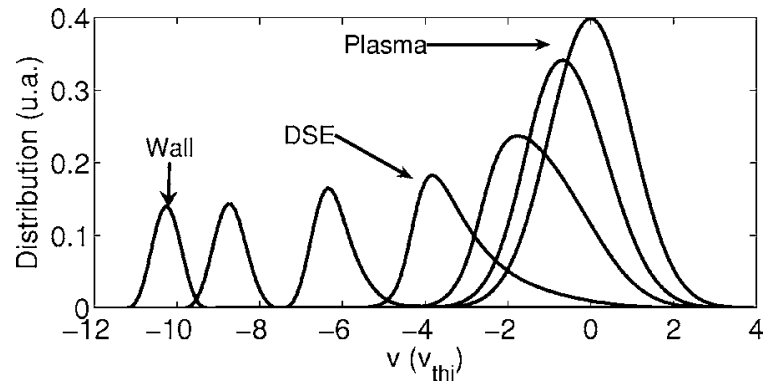

FIG. 12. 1D profiles of the ion velocity distribution for the unmagnetized case, with $\tau=10$ and $\nu=10^{-3}$, for several positions along the plasma-wall transition.

ture peak observed in Fig. 13, which corresponds to the region where the tail of the distribution is most prominent (i.e., the MP).

The physical origin of such a tail in the parallel distribution lies in the competition between collisions, which try to rebuild the equilibrium distribution, and the presence of the wall, which accelerates ions toward it. The ions are accelerated by the electric potential in the CP and the MP, so that their average velocity increases. Therefore, some ions will have a velocity larger (in absolute value) than what may be expected from the equilibrium Maxwellian $f_{0}$. During a collision, these ions will tend, on average, to slow down: the net outcome is to widen the ion distribution function, as shown in Fig. 11(a). Closer to the wall, the acceleration becomes more and more important compared to collisional effects. In the absence of collisions, a potential drop accelerates slower particles more efficiently than faster particles, so that the former will tend to catch up with the latter, leading to a narrowing of the velocity distribution: this is what happens in the DS, where the temperature decreases again. The temperature maximum corresponds to the location where the two competing effects (collisions and acceleration) are of the same order. A similar behavior was observed in previous numerical simulations, ${ }^{19}$ which showed a widening of the $v_{x}$ distribution at the MPE and subsequent narrowing within the DS. Recent experimental studies on unmagnetized plasmas have also confirmed this pattern. ${ }^{22}$

In order to confirm this picture, we performed several runs in the unmagnetized regime (see Fig. 14). In these simulations, the wall is no more at the floating potential, but instead it is polarized to a certain negative potential. By increasing the polarization, we increase the electric field near

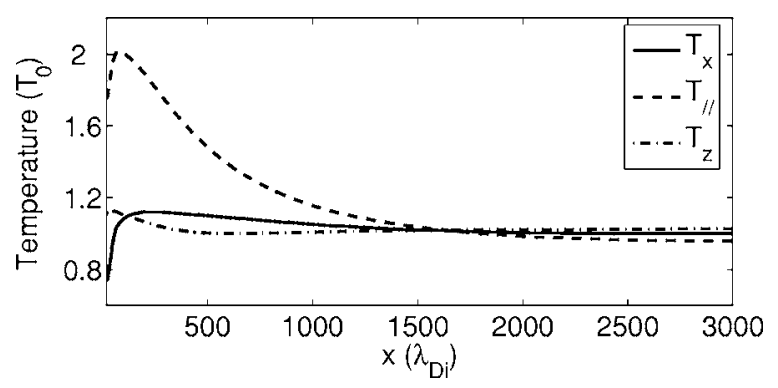

FIG. 13. Temperature profiles for $\alpha=40^{\circ}, \omega=0.010, \tau=10$, and $\nu=10^{-3}$. 


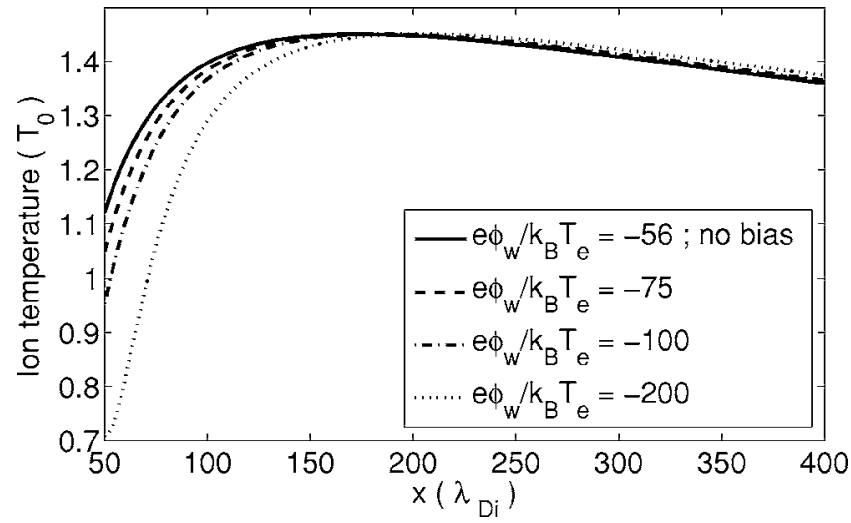

FIG. 14. Temperature profiles for an unmagnetized plasma, with $\tau=10$ and $\nu=10^{-3}$, for different values of the bias applied to the wall.

the wall and thus the extension of the DS. The simulations show that the temperature peak is shifted to the right (i.e., toward the $\mathrm{CP}$ ) as the polarization increases. This result supports our conjecture: for a larger polarization (in absolute value), the effect of the ion acceleration and the corresponding narrowing of the distribution occur at a larger distance from the wall.

Temperature measurements are more meaningful in the direction normal to the wall: as it can be seen in Fig. 11(b), the distribution does not develop a tail along this axis. In the $z$ direction (normal to the magnetic field), the distribution stays even closer to a Maxwellian. Therefore, it appears that the distortion of the velocity distribution is maximum in the direction where the electric field dominates (the parallel direction), whereas it is negligible when the magnetic field dominates (i.e., in the directions normal to $B$ ). The overall variations of the different temperatures (Fig. 13) reflect this behavior: the peak is much less pronounced in the $T_{x}$ temperature and has almost disappeared for the $z$ direction.

The wall has also an important effect on the $E \times B$ drift. In our situation, for which the magnetic field is uniform, this drift is simply proportional to the electric field and directed along the $z$ axis:

$$
\mathbf{v}_{E}=\frac{\mathbf{E} \times \mathbf{B}}{B^{2}}=\frac{E_{x}(x) \cos \alpha}{B} \hat{z} .
$$

We expect the $E \times B$ drift to play an important part in the ion dynamics in the $z$ direction. In order to verify this, we plot in Fig. 15 both the theoretical expression (16) for the $E \times B$ drift and the average velocity along the $z$ axis as measured from the simulations.

The two curves are identical in the $\mathrm{CP}$ and start to differ around $650 \lambda_{\mathrm{D} i}$, which corresponds to the MPE. This discrepancy results from the collection of high-speed ions by the wall. Indeed, the MPE corresponds to the location where the orbits of ions with perpendicular velocity equal to $v_{\text {thi } i}$ intersect the wall. But wall collection is more effective for faster ions (with larger Larmor radii), which are then removed from the distribution, leading to a decrease in the absolute average velocity of the population. Further, in the DS the electric field varies so rapidly that the ion dynamics can no longer be separated into a gyromotion and a drift. A corollary of this

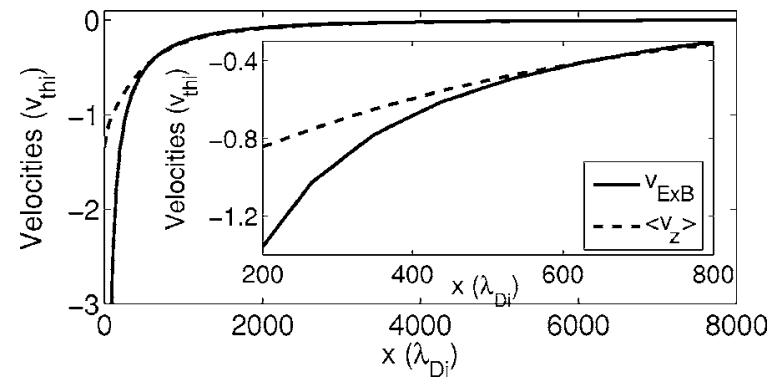

FIG. 15. A comparison between the velocity due to the $E \times B$ drift and the average velocity in the $z$ direction. The inset shows a zoom near the MPE, located at $x=631 \lambda_{\mathrm{D} i}$. This simulation was performed with $\alpha=40^{\circ}$, $\omega=0.010, \tau=10$, and $\nu=10^{-3}$.

result is that approximations based on the guiding center motion (drift-kinetic, gyrokinetic) can only be applied in the CP.

\section{E. Ion distribution at the wall}

An accurate knowledge of the ion distribution at the wall is crucial to determine the effect of the ions on the material surface: ${ }^{23}$ indeed, sputtering and physical adsorption by the surface strongly depend on the energy and angle of incidence of the ions impinging on it.

Here, we focus on the angular distribution $f(\theta)$, where $\theta$ is the angle of incidence, defined as the angle between the velocity vector $\mathbf{v}$ and its projection on the $\left(v_{y}, v_{z}\right)$ plane, as can be see in Fig. 16. This definition of $\theta$ is consistent with that of $\alpha$, the angle of incidence of the magnetic field: $\theta=90^{\circ}$ corresponds to ions impinging on the wall along the normal direction, whereas $\theta \approx 0^{\circ}$ describes ions with a grazing incidence. From Fig. 16, it easily comes that

$$
\theta=\arctan \left(\frac{\left|v_{x}\right|}{\sqrt{v_{y}^{2}+v_{z}^{2}}}\right),
$$

where $v_{x}, v_{y}$, and $v_{z}$ are the components of the velocity vector of an ion striking the wall.

In Figs. 17 and 18, we show the ion distribution functions at the wall for several sets of physical parameters. In each figure, we plot the 2D distribution function in the plane

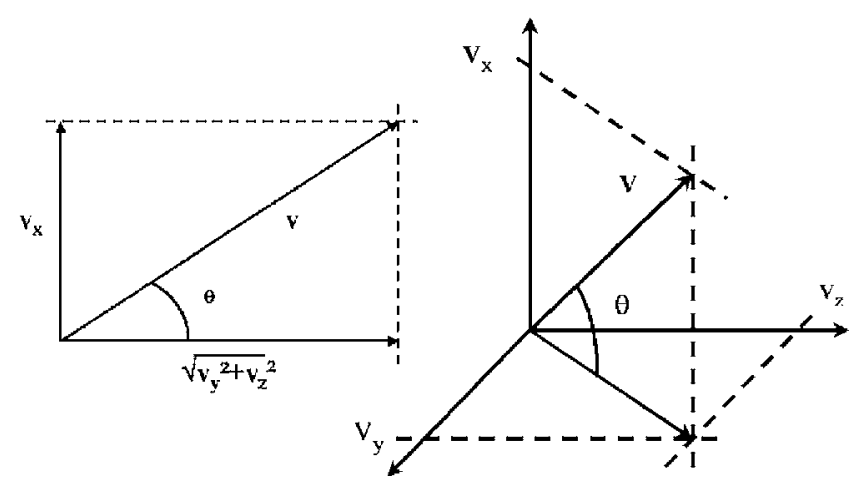

FIG. 16. The angle of incidence $\theta$ is the angle between the velocity vector $\mathbf{v}$ and its projection on the $\left(v_{y}, v_{z}\right)$ plane (where the wall lies). The left part of the figure represents the plane defined by $v_{x}$ and $\mathrm{v}$. Note that this representation is slightly different from that of Fig. 1. 


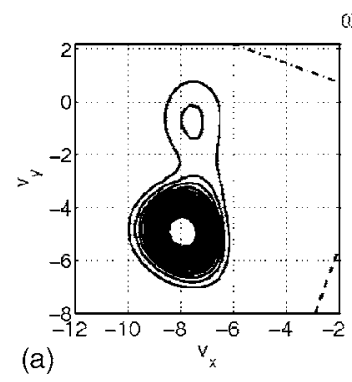

$0-0.100-\alpha-20$

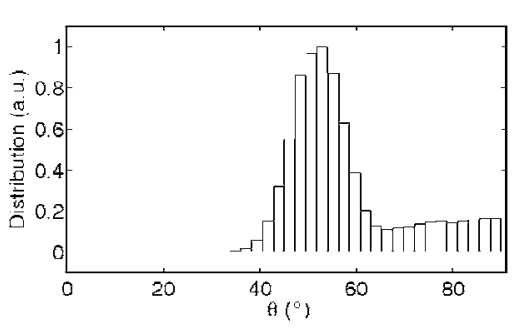

$(g)=0.050 \quad(x=20$
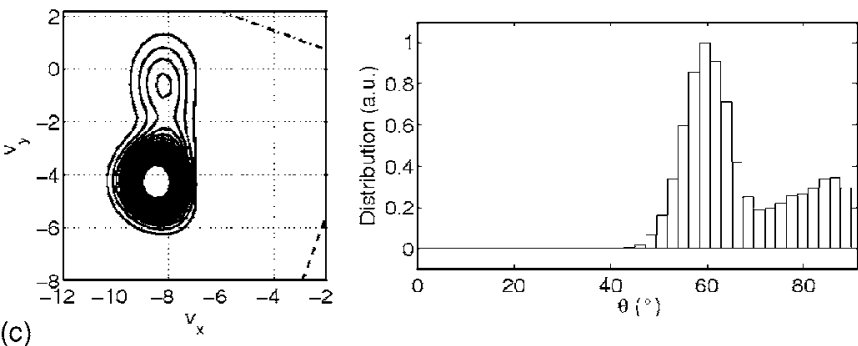

(c)

$$
0-0.010-\alpha-20
$$
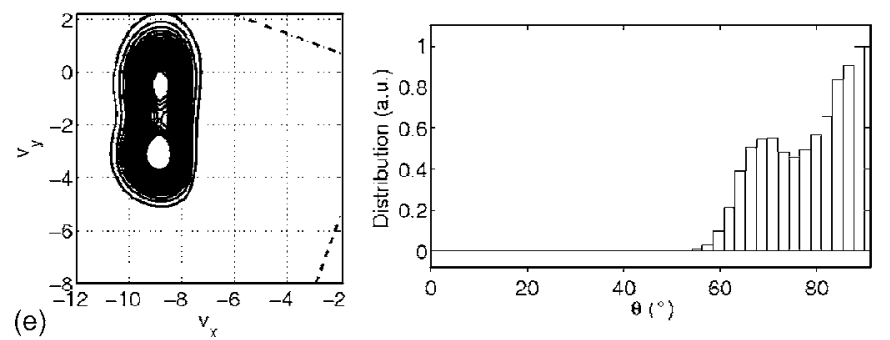

(b)

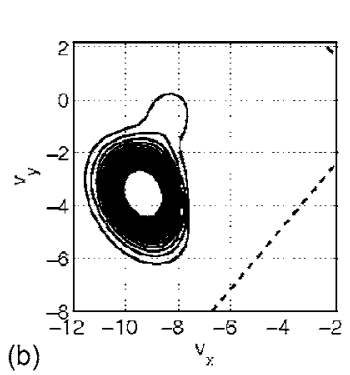

$0=0.100-\alpha=40$

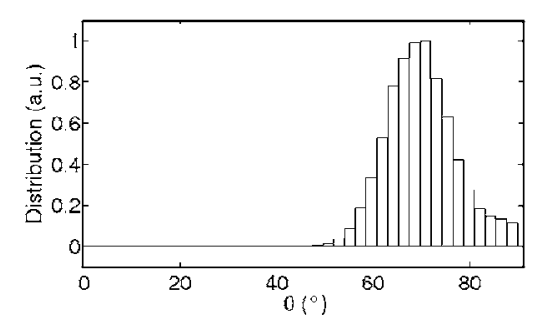

$a=0.050 \quad(\alpha=40$

(d)
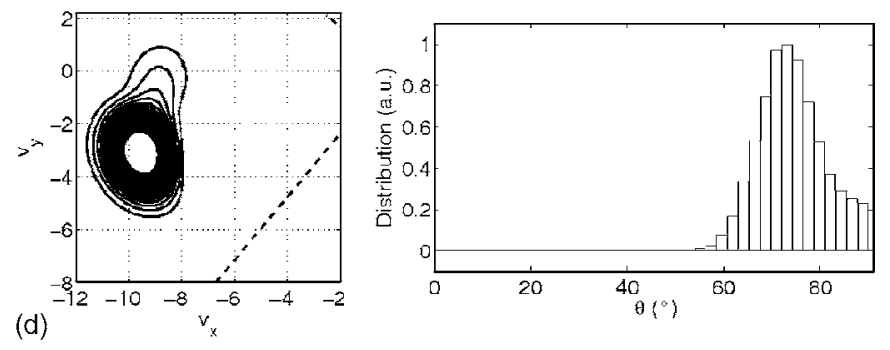

$\omega=0.010-u=40$

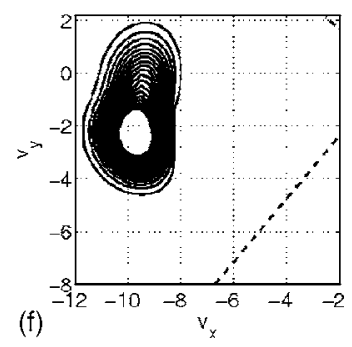

$a=0.010-a=40$

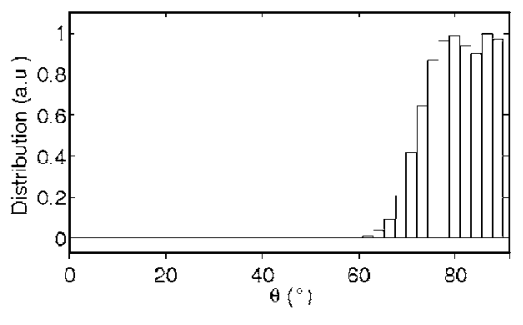

FIG. 17. Ion distribution function on the wall, for various values of $\alpha$ and $\omega$. Left part: contours of the ion distribution at the wall in the $\left(v_{x}, v_{y}\right)$ plane. Right part: angular distribution. The dashed and dash-dotted lines represent, respectively, the directions parallel and perpendicular to the magnetic field. All simulations were performed with $\tau=10$ and $\nu=10^{-3}$.

of the magnetic field $f\left(v_{x}, v_{y}\right)$, and the corresponding angular distribution $f(\theta)$. All simulations of Fig. 17 were performed with a temperature ratio and a collision frequency kept constant at $\tau=10$ and $\nu=10^{-3}$.

We notice that the distribution is sometimes composed of two peaks - see, for instance, Fig. 17(e): the lower peak is centered at a relatively large $v_{y}$ velocity, whereas the upper peak is centered around $v_{y}=0$. The existence of the two peaks can be explained in terms of the competition between the magnetic field and the effects due to collisions.

When the magnetic field is strong compared to the collision rate $(\omega \gg \nu)$, the ions are mainly accelerated along the parallel direction, which entails acceleration toward negative values of $v_{y}$ : this explains the presence of the lower peak in Fig. 17. In the opposite case $(\omega \ll \nu)$, the ions are still accelerated along the field lines, but the isotropizing collisions tend to redirect the ion flow in the direction normal to the wall, so that only one peak around $v_{y}=0$ is visible [see Fig. 8(c) for the case of an unmagnetized transition, $\omega=0]$. For comparable values of $\omega$ and $\nu$, some ions will be accelerated almost without collisions and form the lower peak; other ions will undergo several isotropizing collisions and contribute to the upper peak, thus leading to a distribution of the type observed in Fig. 17(e) (the DS only shifts the distribution in the $v_{x}$ direction, which does not affect the previous conclusions). This effect is clearly visible for $\alpha=20^{\circ}$, but less so for $\alpha=40^{\circ}$, because the parallel direction is closer to the $x$ direc- tion. These two ion populations, because of their different velocities and angles of incidence, may display different behaviors concerning physical adsorption and sputtering at the wall.

In order to validate the above analysis, we performed two more simulations: the first with a large collision rate, $\nu=10^{-2}$, shown in Fig. 18(a); and the second with no collisions $[\nu=0,18(\mathrm{~b})]$. In both cases, we took $\alpha=20^{\circ}$ and $\omega=0.100$. As expected, the peak at $v_{y}=0$ disappears when the collision rate is zero, whereas it becomes dominant when collisions are important. It is interesting to note that the distributions of Figs. 18(a) and 17(e) are very similar to each other, and indeed the ratio $\omega / \nu$ is the same in both cases.

The above phenomenon is reflected in the angular distribution, which also displays two peaks. The peak close to $\theta=90^{\circ}$ corresponds to ions having experienced many collisions, whereas the peak at lower $\theta$ corresponds to ions having been accelerated without collisions along B. In Table I, we give the value of $\theta_{\max }$ for several sets of parameters, $\theta_{\max }$ being the angle corresponding to the maximum of the angular distribution $f(\theta)$. As expected, $\theta_{\max }$ is very sensitive to the value of $\omega$ for $\alpha=20^{\circ}$. It can also be noted that, even with a strong magnetic field $(\omega=0.1), \theta_{\max }$ is still significantly larger than $\alpha$, the angle of incidence of the magnetic field. This difference between $\theta_{\max }$ and $\alpha$ shows that the electric field is always dominant in DS, and manages to redirect the 

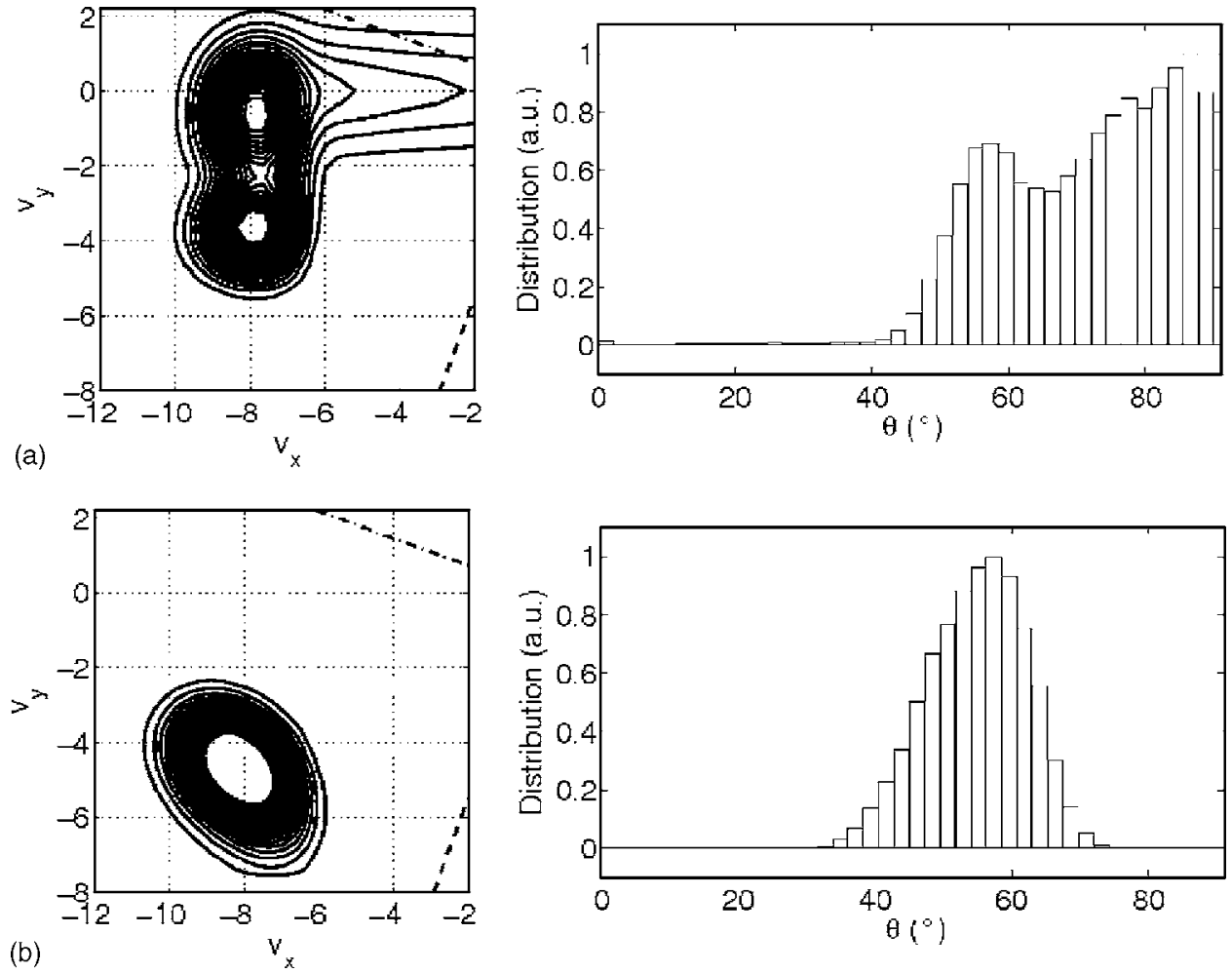

FIG. 18. Ion distribution function in the $\left(v_{x}, v_{y}\right)$ plane and corresponding angular distribution for (a) a large collision rate $\left(\nu=10^{-2}\right)$ and (b) the vanishing collision rate $(\nu=0)$. Other parameters of the simulation are $\alpha=20^{\circ}$, $\omega=0.100$, and $\tau=10$. ions to an angle of incidence closer to the normal to the wall. A similar dependence of $\theta_{\max }$ on $\alpha$ was already suggested by Chodura. $^{24}$

\section{CONCLUSION}

In this paper, we studied plasma-wall interactions in the case of a magnetized and weakly collisional plasma $\left(\lambda_{\mathrm{D} e} \ll \rho_{i} \ll \lambda_{\mathrm{mfp}}\right)$. The physical regimes chosen for the simulations are relevant to low-pressure laboratory plasmas and tokamak edge plasmas. Kinetic Vlasov simulations were performed using an accurate Eulerian code. The use of a nonuniform grid allowed us to simulate the entire transitionfrom the equilibrium plasma to the wall-with a moderate number of grid points. The resulting code enabled us to obtain smooth phase-space distributions along the entire plasma-wall transition region.

The results provided us with an increased understanding of the phase space dynamics of the plasma in the transition region. It is now clear that the plasma is first accelerated along the magnetic field lines in the $\mathrm{CP}$, then is redirected along the axis normal to the wall in the MP, and finally is strongly accelerated in the DS. The thickness of the MP was measured from the simulation results, and was found to be in good agreement with a theoretical estimate.

The ion velocity distribution is considerably deformed in the various sheaths, due to the combined action of the electric and magnetic fields. Therefore, any measure of the ion temperature obtained as the width of the velocity distribution must be taken with care. This deformation is particularly significant in the MP, and corresponds to a spurious peak in the ion temperature. We also showed that the velocity in the direction normal to both the electric and magnetic field is well described by the $E \times B$ velocity in the $\mathrm{CP}$, but departs from it in the MP and even more in the DS.

Finally, we studied the angular distribution of the ions striking the wall. This is an important quantity, as the angle of incidence determines the level of sputtering and erosion of the surface. The results showed that, even for relatively strong magnetic fields, the angle of incidence of the impinging ions is never as grazing as the angle between the magnetic field and the wall. In the regimes considered here, the electric field always manages to partially redirect the ions normally to the surface. Interestingly, we observed that two different ion populations can be present at the wall, giving rise to a two-peak velocity distribution. These two populations correspond to ions that have been drifting without collisions along the magnetic field lines and to ions that have experienced several collisions before hitting the wall. Further work will be necessary to relate more precisely the angular ion distribution to the erosion of the material surface.

TABLE I. Values of $\theta_{\max }$, defined as the angle under which most ions strike the wall, for $\tau=10$ and $\nu=10^{-3}$.

\begin{tabular}{ccccc}
\hline \hline & $\alpha=20^{\circ}$ & $\alpha=40^{\circ}$ & $\alpha=60^{\circ}$ & $\Delta \theta$ \\
\hline$\omega=0.100$ & $52.8^{\circ}$ & $70.8^{\circ}$ & $82.1^{\circ}$ & $\pm 1.25^{\circ}$ \\
$\omega=0.050$ & $59.6^{\circ}$ & $73.1^{\circ}$ & $86.6^{\circ}$ & $\pm 1.25^{\circ}$ \\
$\omega=0.010$ & $70.8^{\circ}$ & $79.8^{\circ}$ & $86.6^{\circ}$ & $\pm 1.25^{\circ}$ \\
$\omega=0.005$ & $78.8^{\circ}$ & $86.6^{\circ}$ & $86.6^{\circ}$ & $\pm 1.25^{\circ}$ \\
\hline \hline
\end{tabular}




\section{ACKNOWLEDGMENTS}

We would like to thank P. Bertrand, J. Bougdira, E. Gravier, S. Heuraux, and R. Hugon for several useful discussions.

${ }^{1}$ P. C. Stangeby, The Plasma Boundary of Magnetic Fusion Devices (Institute of Physics Publishing, London, 2000).

${ }^{2}$ F. Valsaque, G. Manfredi, J. P. Gunn, and E. Gauthier, Phys. Plasmas 9, 1806 (2002).

${ }^{3}$ D. Bohm, in The Characteristics of Electrical Discharges in Magnetic Fields, edited by A. Guthrie and R. K. Wakerling (McGraw-Hill, New York, 1949).

${ }^{4}$ K.-S. Chung and I. H. Hutchinson, Phys. Rev. A 38, 4721 (1988).

${ }^{5}$ K.-U. Riemann, J. Appl. Phys. 24, 493 (1991).

${ }^{6}$ D. Tskhakaya, B. Elliasson, P. K. Shukla, and S. Kuhn, Phys. Plasmas 11, 3945 (2004).

${ }^{7}$ K.-U. Riemann, J. Tech. Phys. 41, 89 (2000).

${ }^{8}$ R. Chodura, Phys. Fluids 25, 1628 (1982).

${ }^{9}$ E. Ahedo, Phys. Plasmas 4, 4419 (1997).
${ }^{10}$ K.-U. Riemann, Phys. Plasmas 1, 552 (1994).

${ }^{11}$ D. Tskhakaya and S. Kuhn, Contrib. Plasma Phys. 44, 564 (2004).

${ }^{12}$ Th. Daube and K.-U. Riemann, Phys. Plasmas 6, 2409 (1999).

${ }^{13}$ D. Sharma, Phys. Plasmas 12, 103506 (2005).

${ }^{14}$ D. Tskhakaya, S. Kuhn, V. Petrzilka, and R. Khanal, Phys. Plasmas 9, 2486 (2002).

${ }^{15}$ C. Z. Cheng and G. Knorr, J. Comput. Phys. 22, 330 (1976).

${ }^{16}$ F. Valsaque and G. Manfredi, Comput. Phys. Commun. 164, 262 (2004).

${ }^{17}$ E. Fijalkow, Comput. Phys. Commun. 116, 319 (1999).

${ }^{18}$ E. Gravier, F. Brochard, G. Bonhomme, T. Pierre, and J.-L. Briançon, Phys. Plasmas 11, 529 (2003).

${ }^{19}$ D. Tskhakaya and S. Kuhn, Europhys. Conf. Abstr. 25A, 1681 (2001); Plasma Phys. Controlled Fusion 47, A327 (2005).

${ }^{20}$ B. Singha, A. Sarma, and J. Chutia, Phys. Plasmas 9, 683 (2002).

${ }^{21}$ J. P. Gunn, Phys. Plasmas 4, 4435 (1997).

${ }^{22}$ G. D. Severn, X. Wang, E. Ko, and N. Hershkowitz, Phys. Rev. Lett. 90, 145001 (2003).

${ }^{23}$ R. J. Seeböck, Surf. Coat. Technol. 166-119, 564 (1999).

${ }^{24}$ R. Chodura, in Physics of Plasma-Wall Interactions in Controlled Fusion, edited by D. E. Post, and R. Behrisch (Plenum, New York, 1986), p. 99. 